\title{
Visualizing Biological Tissues: A Multiscale Workflow from Live Imaging to 3D Cryo-CLEM
}

\author{
Marit de Beer ${ }^{1,2}$, Rona Roverts ${ }^{1,2}$, Xavier Heiligenstein ${ }^{3}$, Edwin Lamers ${ }^{4}$, Nico Sommerdijk ${ }^{1,2}$, $\underline{\text { Anat }}$ \\ Akiva $^{1,2}$ \\ ${ }^{1}$ Department of Biochemistry, Radboud Institute of Molecular Life Sciences, Radboud University \\ Medical Center, Nijmegen, The Netherlands. \\ ${ }^{2}$ Electron Microscopy Center, Radboudumc Technology Center Microscopy, Radboud Institute of \\ Molecular Life Sciences, Radboud University Medical Center, Nijmegen, The Netherlands. \\ ${ }^{3}$ CryoCapCell, Kremlin-Bicêtre, France. \\ ${ }^{4}$ Carl Zeiss BV, Breda, The Netherlands.
}

Cryogenic imaging is a powerful technique to study the ultrastructure of biological tissues as close as possible to their native state, eliminating the risk of image artifacts arising from sample preparation steps such as dehydration, chemical fixation, and heavy metal staining. Cryogenic imaging can also be included in a correlative light / electron microscopy (CLEM) workflow, where the advantages over other CLEM workflows are the high degree of tissue structure preservation, the ability to keep and observe almost all fluorescent probes, and the ability to produce near to native state (CL)EM images.

In the recent years cryo-correlative imaging became a popular approach to investigate proteins and cellular processes in high resolution with cryo-TEM [1] where cryo-FIB/SEM is used as a tool for sample preparation for cryo-TEM analysis. Here, we are extending the correlative workflow combining live imaging, 3D cryo-fluorescence microcopy and 3D cryo-FIB/SEM for high resolution 3D volume imaging, responding to the increasing need to visualize more and more complex systems [2], including organoids [3].

The samples investigated (2D or 3D cell cultures) were grown on a high pressure carrier for the required time (hours to weeks) and were then imaged by live fluorescent microscopy and immediately vitrified using a high-pressure freezer (HPM live- $\mu^{\circledR}$, CryoCapCell) [4]. The frozen sample is then imaged with cryo-fluorescence microscopy using a Linkam stage and a designated holder, to relocate the region of interest for further EM investigation.[4] To increase the localization accuracy between the different instruments, we designed a special flat top high pressure freezing carrier, with a matrix imprint, similar to the well-known TEM finder grids. Upon freezing, the imprint leaves a finder matrix in the upper layer of the vitrified ice that is easily visible both with cryo-light microscopy and cryo-SEM, providing very high accuracy in overlaying the information from the two instruments (figure 1).

3D cryo-FIB/SEM imaging has been demonstrated for soft and hard tissues, cellular organelles, and complex tissue architecture as the paracellular network [5,6]. Samples up to $100 \mu \mathrm{m}$ in depth can be easily imaged using this approach. Moreover, when volume imaging is completed, and higher resolution is required, cryo-lift-out (using a cryo-gripper, Kleindiek) for cryo-TEM imagig can be performed on the last slice of the investigated volume.

This multiscale live-to-cryo CLEM workflow opens a new venue to CLEM imaging, free of artifacts and sample handling.

(c) The Author(s), 2021. Published by Cambridge University Press on behalf of Microscopy Society of America.

This is an Open Access article, distributed under the terms of the Creative Commons Attribution-NonCommercial-NoDerivatives licence (https://creativecommons.org/licenses/by-nc-nd/4.0/), which permits non-commercial re-use, distribution, and reproduction in any medium, provided the original work is unaltered and is properly cited. The written permission of Cambridge University Press must be obtained for commercial re-use or in order to create a derivative work. 


\section{References:}

[1] J Mahamid et al, Science 351 (2016), p. 969.

[2] BWM de Wildt et al, Current Opinion in Biomedical Engineering 10 (2019), p. 107.

[3] A Akiva et al, Advanced Functional Materials 31 (2021), p. 2010524.

[4] X Heiligenstein et al, Methods in Cell Biology, ed. T Müller-Reichert \& P Verkade, Academic Press 162 (2021), p. 115.

[5] N Vidavsky et al, Journal of Structural Biology 196 (2016), p. 487.

[6] A Akiva et al, Journal of Structural Biology 206 (2019), p. 139.

[7] MdB, RR, NS and AA were supported by the European Research Council (ERC) Advanced Investigator grant (H2020-ERC-2017-ADV-788982-COLMIN), AA is also supported by a VENI grant of the Netherlands Scientific Organization NWO (VI.Veni.192.094).

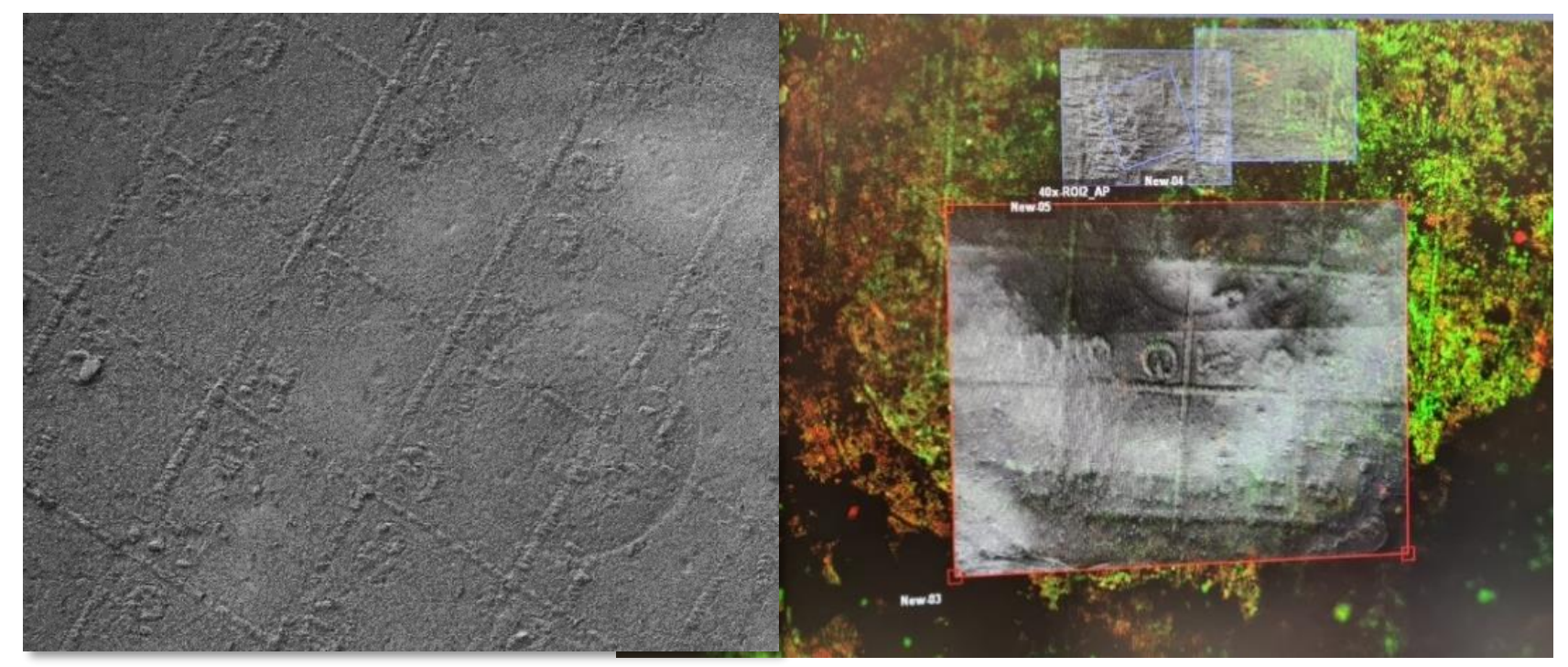

Figure 1. To facilitate the correlation during experiments, we have produced a tailor-made flat top HPF carrier where a matrix is molded into the top layer of the sample. The matrix is visible both in cryo-SEM and cryo fluorescence microscopy and helps to find the ROI with high accuracy. 